\title{
Role of contrast enhanced ultrasound in the assessment of biliary duct disease
}

\section{Zeno Spârchez, Pompilia Radu}

$3^{\text {rd }}$ Medical Department, "Iuliu Hatieganu" University of Medicine and Pharmacy, Institute of Gastroenterology and Hepatology, Department of Ultrasound, Cluj Napoca, Romania

\begin{abstract}
The introduction of microbubble contrast agents (CA), which act as blood pool tracers, has overcome the limitations of conventional B-Mode, colour or power Doppler ultrasound, enabling the display of parenchymal microvasculature. Initially, the use of CA was accepted for hepatic lesions. In the following years, experts have expanded recommendations, as a result of the method's efficiency in extrahepatic applications. In this article we review the methodology and the application of contrastenhanced ultrasound (CEUS) in the evaluation of biliary pathology. This new imaging tool allows a non invasive differential diagnosis of biliary lesions and a clearer delineation of the tumoral process.
\end{abstract}

Keywords: bile duct tumors, ultrasound, contrast enhanced ultrasound

\section{Introduction}

Conventional ultrasound examination (US) has several advantages, such as real-time scanning, easy manipulation, cost-effectiveness, no radiation, high resolution, and repeatability. All these factors have turned this imaging tool into the first-line investigation in the assessment of biliary pathology. Some improvements in the image quality and the introduction of new techniques such as power Doppler, tissue harmonic imaging, 3D imaging and endoscopic ultrasound have increased the diagnostic capabilities of US in the biliary tree [1-3].

In the detection of intrahepatic or extrahepatic bile duct dilatation, ultrasound has up to $100 \%$ sensitivity for experienced examiners [3]. However, this imaging tool lacks the ability to depict microcirculation, an important disadvantage that may lead to failure to establish the nature and the clear delimitation of a biliary tumour.

Received 10.12.2013 Accepted 28.12.2013

Med Ultrason

2014, Vol. 16, No 1, 41-47

Corresponding author: Zeno Spârchez, MD, PhD

$3^{\text {rd }}$ Medical Clinic,

Croitorilor 19-21, Cluj Napoca, Romania

Email:zsparchez@yahoo.co.uk
The microbubble based US contrast agents (USCA) are completely intravascular agents; therefore, their use allows a clear depiction of macro- and micro- circulation of the target lesion, which may lead to an accurate diagnosis. Two important features of these CA should also be mentioned: the non-radioactive character and the use in patients with renal impairment [4]. Although numerous studies have shown promising results of CEUS since 2004, its use in the biliary system was mentioned in the "Guidelines and Good Clinical Practice Recommendation for CEUS" only in the 2011 update [5].

\section{Dose of contrast agent, equipment and technique}

\section{Contrast agents: types and doses.}

The USCA used in daily practice in Europe is SonoVue ${ }^{\circledR}$ (BR1; Bracco SpA, Milan, Italy). The dose depends on the objectives of the study. Therefore, a dose of $2.4 \mathrm{~mL}$ is necessary to characterize a biliary tumour, while in order to depict the biliary tree by intrabiliary administration, only a few drops of SonoVue are recommended [5].

Technique

Each patient needs to be fasted for at least 8 hours before examination. CEUS examination should be pre- 
ceded by a careful assessment of the target lesion with conventional B-mode US. After identification of the target lesion, the transducer is kept in a stable position, while the imaging mode is changed to CEUS, and the mechanical index (MI) settings are adjusted to provide sufficient tissue cancellation, with the maintenance of an adequate depth penetration. A stopwatch is started at the time of SonoVue administration.

For biliary ducts tumour (benign or malignant): SonoVue is administered via the antecubital vein, in a bolus fashion (within 1-2 s), followed by a flush of $10 \mathrm{~mL}$ of $0.9 \%$ normal saline by using a 20 -gauge cannula. The entire CEUS process for the intrahepatic biliary system has to be performed with reference to the adjacent liver parenchyma, starting with arterial (8-30 s from the beginning of CA administration), portal (31-120 s), and finishing with the late (121-360 s) phase. The blood supply of the extrahepatic bile duct is entirely arterial. Therefore, the evaluation needs to be performed in the early phase (10-30 s after contrast injection), as well as in the late phase (31-180 $\mathrm{s}$ after contrast injection) [6].

In the late phase, for patients suspected of malignancy, a liver scanning is mandatory to exclude liver metastasis.

For intracavitary evaluation (biliary ducts): administration is performed through a drainage tube which is inserted into a biliary duct. No standard dosage of UCA has been established for intracavitary injection. The reported range is $0.1 \mathrm{~mL}-1 \mathrm{~mL}$ SonoVue ${ }^{\circledR}$ (most commonly just a few drops) diluted in $20 \mathrm{~mL}$ or more of $0.9 \%$ saline solution. This solution is drawn in a $20-\mathrm{mL}$ injection syringe and slowly injected through the tube [5].

\section{Intrahepatic bile ducts}

\section{Biliary cystadenoma}

Biliary cystadenomas are relatively rare tumours, which tend to occur predominantly in women in their fifth decade. Although it is histologically benign, this tumour has indication for resection, due to its tendency to transform into a cystadenocarcinoma [6].

The typical appearance on US examination is welldefined typically multilocular cystic lesion with a thick wall containing multiple septations or papillary projections.

The CEUS examination in arterial phase may reveal highly echogenic septations, enhancing wall nodules or papillary projections. The enhancement washes out progressively and becomes iso- or hypo-enhancement during the portal and late phases (fig1) [6]. CEUS examination is helpful in evaluating nodule vascularity and facilitates the final diagnosis. Although, it is important to admit that

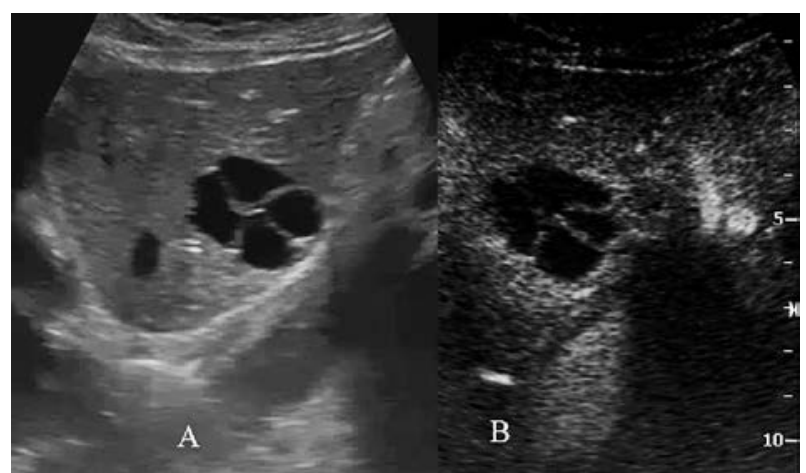

Fig 1. Biliary cystadenoma. A) Transverse US image of the liver of a 55-year-old female with a biliary cystadenoma shows a well-defined structure demonstrating posterior acoustic enhancement and radial septa inside. B) CEUS at $30 \mathrm{~s}$ after the administration of microbubble contrast demonstrates enhancement of the rim component and the septae.

CEUS is not superior to B-scan for the differential diagnosis of cystadenomas and cystadenocarcinomas.

Though it is a rare complication, intracystic bleeding may occur [7]. Due to the secreted mucin, that can mimic intracystic bleeding, the diagnosis of a real intracystic bleeding is very difficult to perform using US alone. According to Naganuma $\mathrm{H}$ et al [7] findings, intracystic bleeding can be diagnosed through the use of CEUS. Unlike a regular examination, the evaluation of the cyst in this case should be performed after 5 minutes from the moment of the intravenous injection of the contrast agent.

\section{Intrahepatic cholangiocarcinoma}

Intrahepatic cholangiocarcinoma (ICC) originates in the small bile ducts. It has a poor prognostic, as a result of the patient's late presentation and lack of effective non-surgical therapeutic modalities [8]. Although ICC is a relatively rare tumour, its worldwide incidence rate has increased steadily in recent years. Since it may develop into cirrhotic livers, it is essential to differentiate between $\mathrm{HCC}$ and ICC.

The US features of ICC are nonspecific. It can appear as a solitary mass with satellite nodules or as diffusely abnormal liver echotexture. The bile ducts peripheral to the tumour mass are usually dilated. Tumour mass may be hypoechoic, hyperechoic, or it may display mixed echogenicity, with irregular borders. Colour Doppler US typically shows a poor colour signal within the tumour.

Imaging findings of ICC showed on CEUS in the arterial phase may be: a) a peripheral irregular rim-like hyperenhancement; b) heterogeneous hypoenhancement and c) heterogeneous hyperenhancement. However, all 


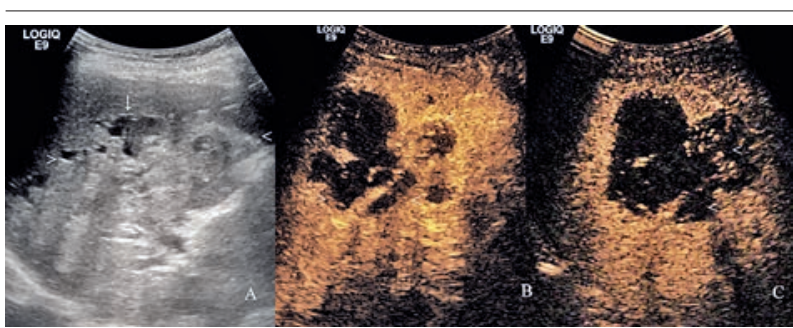

Fig 2. Intrahepatic cholangiocarcinoma. A) Baseline US illustrates an ill-defined echogenic heterogeneous tumor in the fifth segment (arrowhead) with proximal dilatation of the intrahepatic bile ducts (arrow). B) CEUS at $20 \mathrm{~s}$ after microbubble contrast administration demonstrates peripheral irregular rimlike enhancement $(>)$. C) in late phase a obvious wash-out of $\mathrm{CA}$ is demonstrated within the tumour $(>)$.

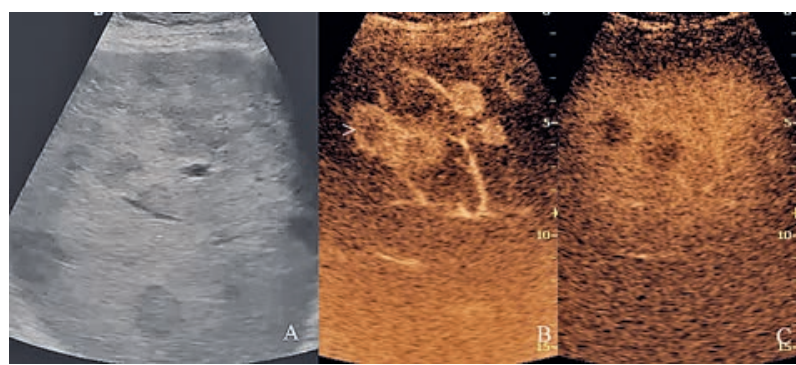

Fig 3. Small multiple intrahepatic cholangiocarcinomas in a 45 old female with VHC liver cirhosis. A) Conventional ultrasound showed multiple hypoecoic lesions in the liver. B) In arterial phase the lesions depict fast rim like hyperenhancing $(>)$. C) In portal phase the lesions wash out becoming marked hypoenhancing.

cholangiocarcinomas show hypoenhancement in the late vascular phase (fig 2) $[6,8,9]$. The enhancement pattern of ICC seemed to change during the course of the tumour's growth. According to Xu HX et al, most ICCs that are less than $3 \mathrm{~cm}$ enhance homogeneously (fig 3), while those over $3 \mathrm{~cm}$ enhance heterogeneously or show a peripherally enhancing rim [6]. Another study showed that the hyperenhancing areas on CEUS exam correspond to abundant carcinoma cell areas. Thus, the authors concluded that CEUS findings of ICC are correlated to the degree of carcinoma's cell proliferation [8]. In a study that compared the contrast enhanced - CT (CECT) exam with CEUS, the accuracy diagnosis was $80 \%$ for CEUS and $67.5 \%$ for CECT. Therefore, CEUS seems to be a new modality for the characterization of ICC [10].

Hepatocellular carcinoma with bile ducts invasion

The portal vein invasion is recognized as a prognostic factor in patients with hepatocellular carcinoma (HCC). Even if it is a rare complication, the bile duct invasion

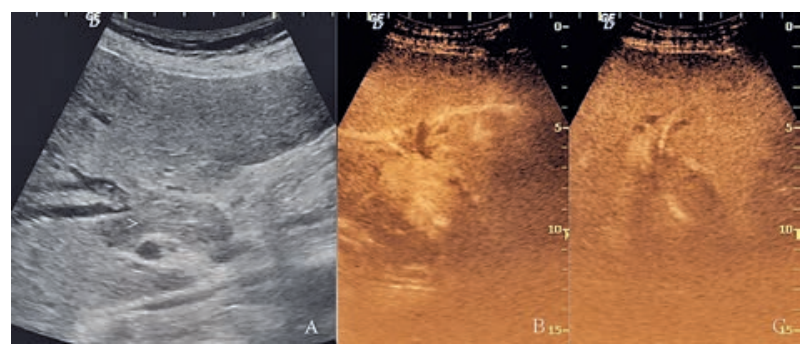

Fig 4. Hypervascular intrabiliary tumor. A) Conventional US shows a $4 \mathrm{~cm}$ long tumor in the main hepatic duct $(>)$; B) in arterial phase the tumor is hyperenhanced in comparison with the surrounding liver parenchyma; C) In venous phase the tumor washes out. Biopsy sampled during ERCP revealed hepatocholangiocarcinoma.

should be considered. The biliary ducts may be affected by direct duct invasion of HCC or by embolisation of HCC in the bile duct, without an obvious parenchymal lesion (icteric-type HCC). Two mechanisms for "icteric type HCC" growth have been proposed: migration of tumour cells into the bile duct and the development of HCC from an ectopic liver parenchyma. The main differential diagnosis of these entities has to be performed with intrahepatic cholangiocarcinoma (including its polypoid variant).

For parenchymal $\mathrm{HCC}$ with invasion of biliary ducts, CEUS examination reveals a homogeneous or heterogeneous hyperenhancement during the arterial phase, as well as washout during the portal or late phase [9].

For the "icteric type HCC", US examination reveals an iso /low-echoic intraluminal polypoid lesion with irregular or smooth surface, depending on the presence of necrosis. CEUS examination shows the characteristic early enhancement pattern of HCC. Because intraductal tumoral thrombi are formed of tumour cells with necrotic materials or a mixture of tumour cells and hematoma, CEUS can demonstrate irregular or sludge-like lesions in the ducts with no enhancement (fig 4).

\section{Hilar cholangiocarcinoma}

This biliary tumour known also as Klatskin's tumour originates at the bifurcation of the hepatic ducts. The lack of symptoms and the difficulties of visualization in an early stage lead to a late diagnosis, thus only less than a half of patients can benefit from surgical resection. In order to establish whether a patient is a candidate for curative resection, an accurate imaging test is mandatory.

The US features of hilar colangiocarcinoma are nonspecific. The first clue and sometimes the only finding is the dilatation of the intrahepatic bile ducts. On conventional US, this tumour may be infiltrative, exophytic or polypoid. Due to the isoechoic appearance, respectively infiltrative pattern of growth, the detection of the tumour 


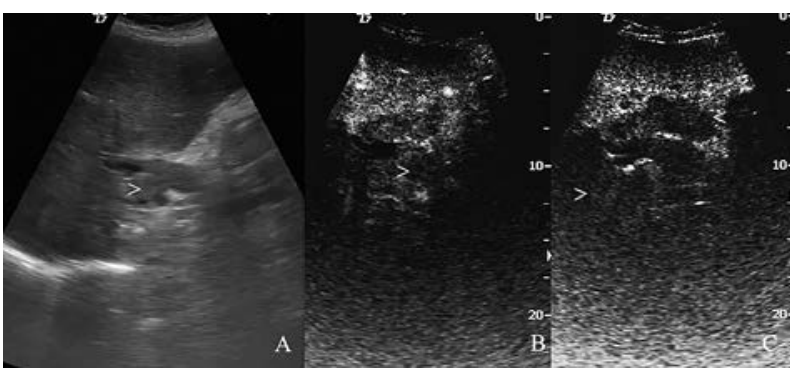

Fig 5. Hilar cholangiocarcinoma. A) B mode US shows a echogenic mass inside the common hepatic duct $(>)$ with infiltration of liver parenchyma. The boundaries of the mass are less defined; B) The lesion ( $>$ ) shows homogeneous hypo-enhancement $26 \mathrm{~s}$ after CA injection on CEUS; C) The lesion is marked hypoenhanced in the late phase. Note that in this phase the margins of the tumor are very well defined $(>)$.

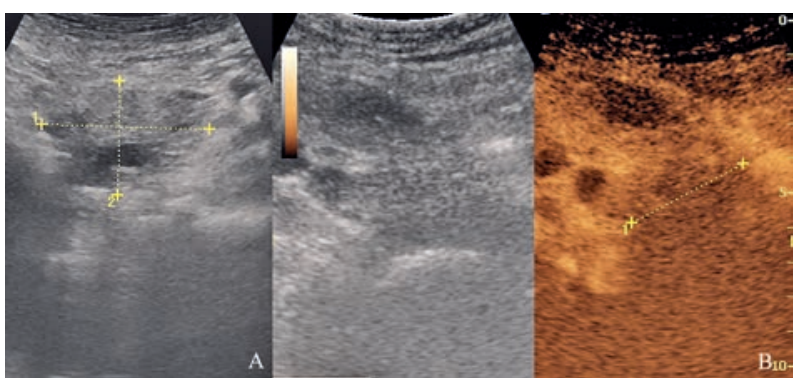

Fig 6. Ampulloma. A) B mode US depicts a hypoechoic mass in the paraduodenal region. B) In arterial phase the lesion shows homogeneous hypo-enhancement.

with its delineating border is sometimes extremely difficult, especially for an inexperienced examiner $[6,11,12]$.

On CEUS examination during the arterial phase, the tumour can appear either with peripheral irregular rimlike hyperenhancement, or as a diffuse heterogeneous hyperenhancement [12]. As enhancement the tumours are hyper-enhanced, isoenhanced and hypo-enhanced in $43.8 \%, 43.8 \%$, and $12.4 \%$ of cases [6]. In the late phase, the tumour appears hypoenhanced, in comparison with adjacent enhanced liver parenchyma, thus the tumour border is well delineated and the infiltration extent is well depicted (fig 5) The enhancement features are related to the histological characteristics of the tumour and CA (blood pool agent). Pathologically, $70 \%$ of tumours are sclerosing adenocarcinomas, with mass of fibrous tissue hyperplasia around the gland cavity [11].

According to Xu HX et al, through the use of CEUS, the conspicuity of the tumour has been defined as definitely distinct in $93.8 \%$ cases, which was a significantly higher rate than in the case of the CECT examination (78.1\%). Also, through the use of this method, the diag- nosis accuracy for portal vein or hepatic duct invasion has increased up to $90 \%$ [11].

\section{Extrahepatic biliary ducts}

\section{Villous adenoma}

Villous adenomas are benign epithelial lesions with malignant potential, rarely encountered in the biliary tree. It is difficult to differentiate villous adenoma in the extrahepatic bile duct from biliary sludge, since they have similar echogenicity on US examination.

On CEUS examination in the arterial phase, the mass shows homogeneous enhancement. In the late phase, the enhancement decreases gradually, so that the mass becomes hypo-enhanced towards the end of the late phase [6].

\section{Ampullary carcinoma}

Ampullary tumours are relatively uncommon, representing only 1.5 per cent of gastrointestinal tract tumours. Due to the localization of ampulla, the role of US in the diagnosis of ampullary carcinoma is low. So, in general, on US examination, indirect signs such as bile duct or pancreatic duct dilatation are visualized.

On CEUS examination in the arterial phase, the tumour is iso-enhanced and becomes hypo-enhanced in the late phase (fig 6). According to Kiura et al, the type of enhancement of ampullary tumour may offer supplementary information regarding the growth pattern [13]. The enhancement in arterial phase may differentiate this tumour from other lesions arising at this level, such as hypervascular GISTs or neuroendocrine tumours.

\section{Intrabiliary metastasis}

Intrabiliary metastases are rare, and their imaging features make them easy to confuse with primary biliary tumours, especially with cholangiocarcinoma. The primary tumours known as causing metastatic biliary obstruction include colonic cancer, malignant melanoma, lymphoma, gallbladder, ovary, duodenum, oesophagus, liver, cervix, uterus, muscle, prostate, bone, and brain. Most often, the intrabiliary metastasis originates in colonic carcinoma.

On US examination, these tumours often appear as solid masses associated with dilated peripheral ducts. For a right diagnosis, two steps are essential: to determine the nature of the intrabiliary lesion (benign or malignant) and to establish the type of malignant tumour (cholangiocarcinoma, HCC or metastasis).

In a study conducted by Lee YJ, there is a difference of enhancement between intraductal metastasis and cholangiocarcinoma. Thus, intraductal metastasis tends to exhibit hypervascularity in the arterial phase, whereas intraductal cholangiocarcinoma has a tendency towards isoattenuation (fig 7) [14]. 


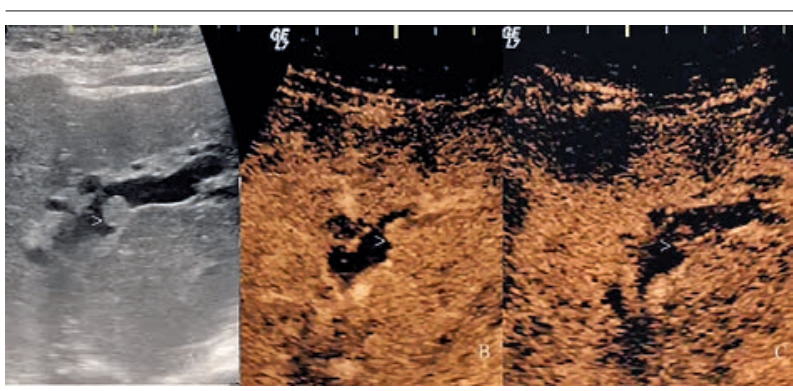

Fig 7. Intrabiliary metastasis in a patient with colic cancer and hepatic metastases. A) B mode US reveals an echogenic round small lesion in the left hepatic duct $(>)$. B) In arterial phase the lesion shows homogeneous hyper-enhancement; C) in the late-phase the lesion washes-out in respect with the surrounding liver.

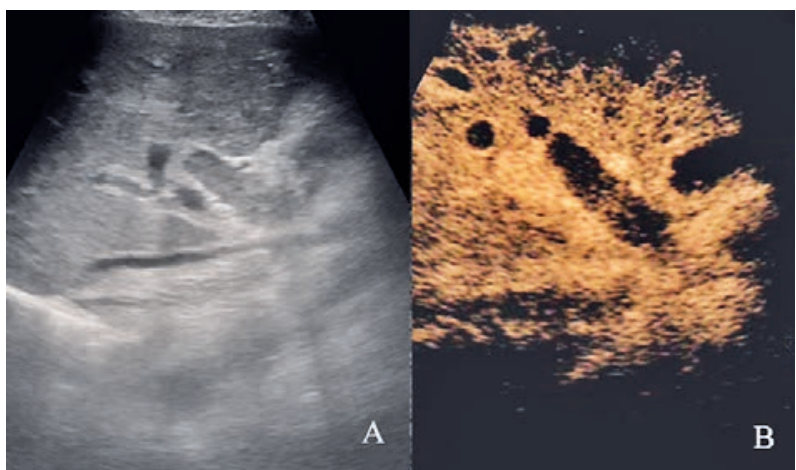

Fig 8. A) Echogenic mass in common bile duct in a female patient with haemobilia. B) In arterial phase the lesion does not show any enhancement confirming the nontumoral origin.

Nontumoral intrabiliary masses like pus, clots, parazites, sludge or nonshadowing stones can be differentiated with high accuracy from tumors by means of CEUS (fig 8).

\section{Non tumoral biliary pathology}

\section{Portal hypertensive biliopathy (PHB)}

Portal hypertensive biliopathy is defined as an abnormality of the walls of the biliary tree secondary to portal hypertension. It is usually diagnosed incidentally; however, a small percentage of patients develop symptomatic bile duct obstruction. The drainage veins of the common bile duct form a pericholedochal and paracholedochal venous plexus. Hypertrophy of both collateral plexuses produces mural irregularities, and sacular dilatation, which can progress to stenosis. The main differential diagnosis is with cholangiocarcinoma.

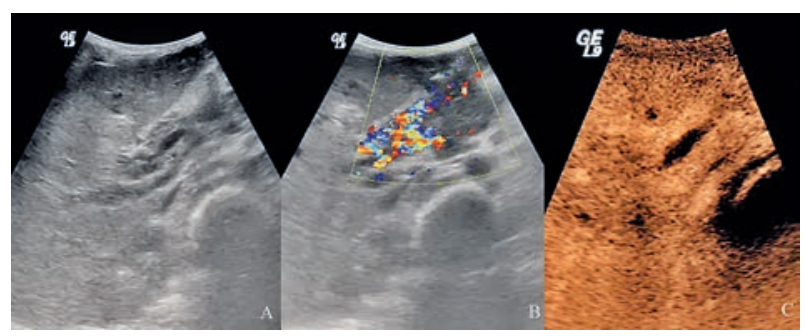

Fig 9. A) Diffuse thickening of the common bile duct in a female patient with portal vein thrombosis (>); B) Color Doppler US shows numerous tortuous vessels in the wall. C) In portal phase the walls become hyperenhanced $(>)$.

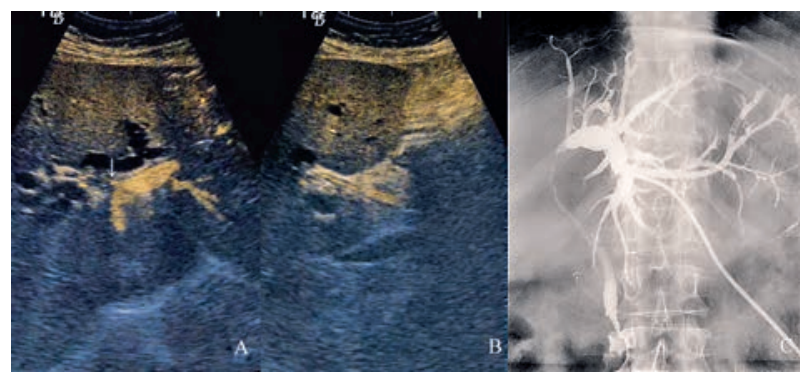

Fig 10. Klatskin tumor. Percutaneous biliary drainage in the left lobe; A) CEUS cholangiography shows enhancement of the left posterior intrahepatic bile ducts with a narrow stenosis at the level of left hepatic bile duct $(\downarrow)$. No communication with anterior IHBD and right bile duct is seen; B) the presence of CA into the common bile duct as well as the depiction of a stenosis are demonstrated $(<)$; C) Rx cholangiography reveals a stenosis of the LHD and CHD with no communication to the right biliary tree

US examination reveals a thickened wall of the common bile duct. In $35 \%$ of patients with PHB, gallbladder varices are also described [15].

On CEUS examination after SonoVue injection, the paracholedocheal veins become enhanced, and appear as beads. After several seconds, the pericholedochal veins become linearly enhanced. Thereafter, the whole bile ducts gradually become enhanced (fig 9). Using this imaging method, a clear differentiation between the dilatation of paracholedocheal veins and pericholedocheal venous plexuses can be distinguished [16].

\section{Periductal inflammation}

Inflammation of the bile ducts may occur in acquired immunodeficiency syndrome (AIDS) patients, owing to an opportunistic infection or in patients with intrahepatic cholelithiasis. 
On US examination, a wall thickening of the bile duct and hypoechogenicity around the intrahepatic bile duct may appear.

CEUS examination shows hyper-enhancement during the arterial phase and hypo-enhancement during the late phase [6].

\section{Contrast agent injection into biliary ducts}

\section{Bile duct drainage}

With accuracy between $96 \%$ and $100 \%$ in detecting biliary obstruction, $\mathrm{X}$-ray percutaneous transhepatic cholangiography (PTC) was considered once the standard method to evaluate the level of obstruction $[17,18]$. In spite of this, it has several important disadvantages: it is radioactive and inappropriate for patients that are sensitive to iodine. Another drawback is the fact that PTC had an accuracy of only $63 \%$ in determining the cause of obstruction [19]. According to the latest reports, percutaneous ultrasound cholangiography (PUSC) provides visualization of the bile ducts as small as fifth-order branches [20]. Regarding the accuracy of PUSC in determining the level and the cause of obstruction, Luyao $\mathrm{Z}$ et al concluded that this new method is comparable to PTC and may be used as an alternative method to X- ray PTC (fig 10) [19].

Percutaneous transhepatic biliary drainage (PTBD) is an alternative method for the palliative treatment of a malignant biliary obstruction. In order to ensure the effectiveness of the drainage, it is critical to identify the tip of the drainage catheter. Drainage tubes can be detected by US, but they can hardly be seen in their full length and the tip position is often difficult to locate. In 2012 Xu EJ et al, demonstrated that PUSC improved the visualization of the drainage catheters and location of their tips. Also, the diagnostic accuracy of PUSC for biliary obstruction level was as high as $100 \%$ [21].

\section{Biliary leakage}

Until 2010, PTC was the preferred examination for diagnosis of biliary leakage due to its high sensitivity and anatomic resolution. However, due to the above mentioned disadvantages other possible applications of PUSC have emerged. These evaluate possible complications after $\mathrm{T}$ tube removal (biliary leakage) and liver transplantation (anastomotic). For the diagnosis of leakage or stenosis, X ray PTC has proven to be more accurate than PUSC. The main cause was the presence of gas inside the bowels. By contrast, the image quality of the intrahepatic bile ducts regarding the visualization of ductal branches was comparable to X ray PTC [22]. In 2010, Mao R et al, demonstrated the utility of PUSC in diagnosis of biliary leakage after $\mathrm{T}$ tube removal [23].

\section{Conclusions}

CEUS is a complementary method that can be utilized for supplementary evaluation of US findings. The summarized indications for evaluating biliary ducts with CEUS are: (1) to perform a differential diagnosis between intrahepatic cholangiocarcinoma and other tumors (hepatocellular carcinoma or liver metastasis), (2) to characterize the biliary cystadenoma and diagnose complications; (4) to depict the borders of Klatskin's tumor with greater clarity; (5) to make a distinction between polypoid cholangiocarcinoma and intrabiliary metastasis; (6) to make a distinction between cholangiocarcinoma and benign ductal pathology (periductal inflammation or portal biliopathy); (7) to differentiate common bile duct cancers from nontumoral intrabiliary lesions ( pus, blood, sludge ); 8) to identify the tip location of the drainage catheter and the presence of biliary leakage.

This imaging method is particularly useful for those patients for whom the use of contrast agents in CT or MR imaging is contraindicated (renal impairment and allergy to CA). It is also important to highlight the fact that limitations encountered in US examination are not totally eliminated by CEUS examination.

\section{Conflict of interest: none}

\section{References}

1. Ortega D, Burns PN, Hope Simpson D, Wilson SR. Tissue harmonic imaging: is it benefit for bile duct sonography? AJR Am J Roentgenol 2001; 176: 653-659.

2. Xu HX, Yin XY, Lu MD, Liu L, Yue DC, Liu GJ. Comparison of three- and two-dimensional sonography in diagnosis of gallbladder diseases: preliminary experience. J Ultrasound Med 2003; 22: 181-191.

3. Kawashima H, Hirooka Y, Itoh A, et al. Progress of endoscopic ultrasonography and intraductal ultrasonography in the diagnosis of malignant biliary diseases. J Hepatobiliary Pancreat Surg 2006; 13: 69-74.

4. Rösch T, Meining A, Frühmorgen S, et al. A prospective comparison of the diagnostic accuracy of ERCP, MRCP, $\mathrm{CT}$, and EUS in biliary strictures. Gastrointest Endosc 2002; 55: 870-876.

5. Piscaglia F, Nolsøe C, Dietrich CF, et al. The EFSUMB Guidelines and Recommendations on the Clinical Practice of Contrast Enhanced Ultrasound (CEUS): update 2011 on non-hepatic applications. Ultraschall Med 2012; 33: 33-59.

6. Xu HX. Contrast-enhanced ultrasound in the biliary system: Potential uses and indications. World J Radiol 2009; 1: $37-44$.

7. Naganuma H, Funaoka M, Fujimori S, et al. Contrastenhanced sonographic findings in a case of hepatobiliary 
cystadenoma with intracystic bleeding. J Clin Ultrasound 2006; 34: 412-415.

8. Xu HX, Chen LD, Liu LN, Zhang YF, Guo LH, Liu C. Contrast-enhanced ultrasound of intrahepatic cholangiocarcinoma: correlation with pathological examination. Br J Radiol 2012; 85: 1029-1037.

9. Chen LD, Xu HX, Xie XY, et al. Intrahepatic cholangiocarcinoma and hepatocellular carcinoma: differential diagnosis with contrast-enhanced ultrasound. Eur Radiol 2010; 20: 743-753.

10. Chen LD, Xu HX, Xie XY, et al. Enhancement patterns of intrahepatic cholangiocarcinoma: comparison between contrast-enhanced ultrasound and contrast-enhanced CT. Br J Radiol 2008; 81: 881-889.

11. Xu HX, Chen LD, Xie XY, et al. Enhancement pattern of hilar cholangiocarcinoma: contrast-enhanced ultrasound versus contrast-enhanced computed tomography. Eur J Radiol 2010; 75: 197-202.

12. Meacock LM, Sellars ME, Sidhu PS. Evaluation of gallbladder and biliary duct disease using microbubble contrast-enhanced ultrasound. Br J Radiol 2010; 83: 615-627.

13. Kiura N, Okamura S, Urano F, et al. Contrast-enhanced US of ampullary carcinoma: correlations with pathological findings. J Med Ultrasonics 2012; 39: 53-59.

14. LeeYJ, Kim SH, Lee JY, et al. Differential CT features of intraductal biliary metastasis and double primary intraductal polypoid cholangiocarcinoma in patients with a history of extrabiliary malignancy. AJR Am J Roentgenol 2009; 193: 1061-1069.
15. Chattopadhyay S, Nundy S. Portal biliopathy. World J Gastroenterol 2012; 18: 6177-6182.

16. Nunoi H, Hirooka M, Ochi H, et al. Portal biliopathy diagnosed using color Doppler and contrast-enhanced ultrasound. Intern Med 2013;52:1055-1059.

17. Koenigsberg M, Wiener SN, Walzer A. The accuracy of sonography in the differential diagnosis of obstructive jaundice: a comparison with cholangiography. Radiology 1979; 133: $157-165$

18. Goldstein LI, Sample WF, Kadell BM, Weiner M. Gray-scale ultrasonography and thin-needle cholangiography. Evaluation in the jaundiced patient. JAMA 1977; 238: 1041-1044.

19. Luyao Z, Xiaoyan X, Huixiong X, Zuo-Feng X, GuangJian L, Ming-de L. Percutaneous ultrasound-guided cholangiography using microbubbles to evaluate the dilated biliary tract: initial experience. Eur Radiol 2012; 22: 371-378.

20. Roberts JP, Neill A, Goldstein R. The use of a micro-bubble contrast agent to allow visualization of the biliary tree. Clin Transplant 2006; 20: 740-742.

21. Xu EJ, Zheng RQ, Su ZZ, Li K, Ren J, Guo HY. Intra-biliary contrast-enhanced ultrasound for evaluating biliary obstruction during percutaneous transhepatic biliary drainage: a preliminary study. Eur J Radiol 2012; 81: 3846-3850.

22. Chopra SS, Eisele R, Stelter L, et al. Contrast enhanced ultrasound cholangiography via T-tube following liver transplantation. Ann Transplant 2012; 17: 108-112.

23. Mao R, Xu EJ, Li K, Zheng RQ. Usefulness of contrastenhanced ultrasound in the diagnosis of biliary leakage following T-tube removal. J Clin Ultrasound 2010; 38: 38-40. 